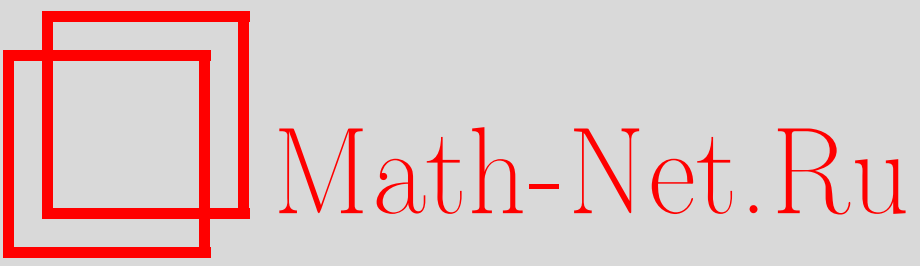

М.-М. Деза, М. И. Штогрин, Бесконечные примитивные полициклы, УМН, 2000, том 55, выпуск 1, 179-180

DOI: https://doi.org/10.4213/rm253

Использование Общероссийского математического портала Math-Net.Ru подразумевает, что вы прочитали и согласны с пользовательским соглашением

http://www.mathnet.ru/rus/agreement

Параметры загрузки:

IP : 54.92 .164 .108

26 апреля 2023 г., 14:15:50 


\title{
БЕСКОНЕЧНЫЕ ПРИМИТИВНЫЕ ПОЛИЦИКЛЫ
}

\author{
М. ДЕзА, М. И. Штогрин
}

В работе [1] для конечного планарного граффа $G$, обладающего некоторым специальным внутренним устройством, а также некоторой специальной укладкой на плоскости, определено понятие односвязного примитивного поли- $r$-цикла $\Pi(G)$ и доказаны две важные теоремы, касающиеся этого понятия. Ниже обе теоремы обобщены на случай бесконечного графа.

СоглАшениЕ. У бесконечного графа имеется счетное число вершин, в каждой вершине сходится конечное число ребер, все эти числа ограничены в совокупности. Любая конечная область на плоскости содержит конечное число вершин бесконечного плоского графа, непустое пересечение двух ребер есть только вершина, бесконечных вершин нет. Области, определяемые бесконечным плоским графом, назовем его гранями, причем ограниченную область назовем внутренней гранью, а неограниченную область назовем внешней гранью.

ОПредЕЛЕнИЕ. Бесконечный плоский граф $G$ вместе со всеми его внутренними гранями назовем $n o л u-r-u$ иклом и обозначим через $\Pi(G)$, если выполнены следующие три условия:

(1) граф̆ $G$ неразделим (при удалении любой его вершины $v$ графф $G-v$ остается связным);

(2) все внутренние грани графа $G$ ограничены простыми циклами одной и той же длины $r$;

(3) пересечение любых двух внутренних граней графа $G$ есть либо одно ребро, либо пусто.

ЗАмечАниЕ. Сформулированное здесь условие (1) универсально: оно годится не только для бесконечного графа, но и для конечного графа, см. [1], откуда взяты условия (2) и (3). Внутреннюю грань плоского графа $G$ впредь будем называть гранью поли- $r$-цикла $\Pi(G)$.

Полицикл $\Pi(G)$ лежит в плоскости и представляет собою односвязную область. В самом деле, если бы в полицикле была дыра, ее окружал бы некоторый простой цикл из графа $G$. По теореме Жордана простой цикл ограничивает некоторую конечную область. По заключенному соглашению в конечной области содержится конечное число вершин (и ребер) бесконечного графа. Поэтому область представляет собою объединение конечного числа $(\geqslant 1)$ внутренних граней. Значит, никаких дыр в ней нет. В силу условия (3) в каждой вершине сходится не более трех граней полицикла. Ровно три грани составляют одну циклическую последовательность вокруг внутренней вершины полицикла. Меньше трех граней полицикла входят в одну циклическую последовательность вокруг граничной вершины полицикла вместе с одной (при наличии двух внешних граней, инцидентных с одной вершиной, удаление вершины нарушило бы связность графа) внешней гранью. Поэтому с граничной вершиной инцидентны либо две грани полицикла, смежные по ребру, либо только одна грань.

Теорема 1. Если бесконечный планарный зраф допускает укладку на плоскости, реализующую поли-r-цикл $\Pi(G)$, определяемый условиями (1)-(3), то только одну.

В самом деле, в силу условия (2) грани поли- $r$-цикла $\Pi(G)$ суть $r$-угольники. В силу следствия из основной леммы грани ограничены кратчайшими реберными циклами из граффа $G$. Поэтому комбинаторное устройство полицикла П $(G)$ однозначно определяется графом $G$.

Пусть $\left(r^{3}\right)$ - разбиение сферы $\mathbb{S}^{2}$ при $r=3,4,5$, евклидовой плоскости $\mathbb{R}^{2}$ при $r=6$ и плоскости Лобачевского $\mathbb{H}^{2}$ при $r \geqslant 7$ на правильные $r$-угольники с углами $\frac{2 \pi}{3}$ при вершинах. Каждому правильному разбиению $\left(r^{3}\right)$ или поли- $r$-циклу П $(G)$ однозначно сопоставим комбинаторно изоморфиный ему абстрактный двумерный полиэдр $\mathbf{K}\left(r^{3}\right)$ или $\mathbf{P}(G)$ соответственно, составленный из правильных евклидовых $r$-угольников.

Назовем поли- $r$-цикл $\Pi(G)$ собственныц, если граф $G$ является частичным подграфом реберного остова правильного разбиения $\left(r^{3}\right)$, и несобственным в противном случае. Несобственный поли- $r$-цикл $\Pi(G)$ назовем гелиценом над $\left(r^{3}\right)$, если существует комбинаторное отображение $\Pi(G) \rightarrow\left(r^{3}\right)$, при котором не менее двух ребер имеют один и тот же образ.

Работа выполнена при поддержке Российского фонда фундаментальных исследований (грант № 99-01-00010). 
ОСНОВНАЯ ЛЕМмА. Пусть П $(G)$ есть произвольный бесконечный односвязный примитивный поли-r-цикл. Тогда существует непрерывное локально изометрическое отображение $f: \mathbf{P}(G) \rightarrow \mathbf{K}\left(r^{3}\right)$, сохраняющее клеточную структуру полиэдра $\mathbf{P}(G) \sim \Pi \mathbf{\Pi}(G)$.

Если $f$ является топологическим отображением полиэдра $\mathbf{P}(G)$ на его образ в $\mathbf{K}\left(r^{3}\right)$, то полицикл $\boldsymbol{\Pi}(G)$ собственный. Если отображение $f$ полиэдра $\mathbf{P}(G)$ на его образ в $\mathbf{K}\left(r^{3}\right)$ является не топологическим отображением, а гомоморфизмом, то полицикл $\Pi(G)$ является гелиценом над $\left(r^{3}\right)$. Эти два варианта исчерпывают все возможности для отображения $f$.

ТЕОрема 2. Любой несобственный бесконечный односвязный примитивный поли$r$-цикл $\Pi(G)$ является гелиценом над $\left(r^{3}\right)$. Комбинаторное отображение $\Pi(G)$ в $\left(r^{3}\right)$ единственно.

СЛЕДСТВИЕ ИЗ ОСНОВНоЙ ЛЕММЫ. В любом бесконечном односвязном примитивном поли-r-цикле $\Pi(G)$ произвольный $r$-цикл графа $G$ ограничивает грань данного поличикла.

Лишш в трех исключительных случаях $\Pi\left(3^{3}\right) \sim \mathbf{P}\left(3^{3}\right)=\mathbf{K}\left(3^{3}\right)-\mathbf{f}, \mathbf{\Pi}\left(4^{3}\right) \sim \mathbf{P}\left(4^{3}\right)=$ $\mathbf{K}\left(4^{3}\right)-\mathbf{f}, \mathbf{\Pi}\left(5^{3}\right) \sim \mathbf{P}\left(5^{3}\right)=\mathbf{K}\left(5^{3}\right)-\mathbf{f}$, имеется один $r$-цикл, ограничиваюший удаленную из многогранника грань $\mathbf{f}$. Любой другой $r$-цикл в этих трех случаях, $r=3,4,5$, а также любой $r$-цикл в любом другом поли- $r$-цикле П $(G)$ ограничивает грань полицикла, см. [1].

ДокАЗАТЕЛЬСТво основной лЕмМы. Отдельный $r$-угольник полиэдра $\mathbf{P}(G)$ допускает изометрическое отображение на $r$-угольник полиэдра $\mathbf{K}\left(r^{3}\right)$, так как полиэдры $\mathbf{P}(G)$ и $\mathbf{K}\left(r^{3}\right)$ состоят из одинаковых евклидовых правильных $r$-угольников. Отображение одного $r$-угольника может быть однозначно продолжено до отображения соседнего $r$-угольника, смежного с ним по стороне. Любые два $r$-угольника полиэдра $\mathbf{P}(G)$ можно соединить цепочкой $r$-угольников, в которой первый и последний $r$-угольники совпадают с двумя данными, и любые два соседние $r$-угольника цепочки смежны по стороне. В самом деле, пусть простой путь $A B$ в реберном графе соединяет вершины $A$ и $B$ каких-либо двух $r$-угольников полиэдра $\mathbf{P}(G)$. Достаточно малым шевелением пути $A B$ в полиэдре $\mathbf{P}(G)$ обойдем каждую встречную вершину, если не слева, то справа, что всегда можно сделать, так как иначе граф $G$ был бы лишь 1-связным, а не 2-связным. Полученному пути соответствует нужная нам цепочка. По этой цепочке отображение первого $r$-угольника однозначно продолжается до отображения последнего. Покажем, что это отображение не зависит от выбора цепочки.

Замкнутую цепочку $r$-угольников из $\mathbf{P}(G)$ назовем әлементарной, если она состоит из

$(\alpha)$ двух смежных по стороне $r$-угольников, с переходом от одного к другому и обратно;

$(\beta)$ трех сходящихся во внутренней вершине $r$-угольников, с полным обходом вокруг нее.

Полиэдры $\mathbf{P}(G)$ и $\mathbf{K}\left(r^{3}\right)$ не только состоят из равных правильных $r$-угольников, они обладают изометричными парами смежных по стороне $r$-угольников, тройками сходящихся в вершине $r$-угольников (когда полиэдр $\mathbf{P}(G)$ имеет внутреннюю вершину). Как изометрия пары, так и изометрия тройки однозначно определяется по своему ограничению на одном $r$-угольнике: после обхода вдоль элементарной замкнутой цепочки мы снова окажемся в исходном $r$-угольнике вместе с исходным его изометрическим отображением.

Опять вернемся к произвольной цепочке. Если в каком-либо месте этой цепочки мы вставим или выбросим элементарную замкнутую цепочку, то при таком (элементарном) преобразовании цепочки отображение, соответствующее новой цепочке, останется прежним. А так как с помощью элементарных преобразований любую цепочку, соединяющую фиксированные два $r$-угольника, можно перевести в любую другую такую цепочку, что следует из односвязности полиэдра $\mathbf{P}(G) \sim \boldsymbol{\Pi}(G)$, то отображение не зависит от выбора цепочки. Итак, отображение одного $r$-угольника порождает отображение всего полиэдра. Лемма доказана.

СПИСОК ЛИТЕРАТУРЫ

[1] Деза М., Штогрин М.И.// УМН. 1999. Т. 54. №6. С. 159-160.

LIENS, Ecole Normale Supérieure and CNRS, Paris; Математический институт им. В. А. Стеклова РАН 